\title{
Relaciones comerciales de Perú y América Latina en contextos de cambios del mercado internacional: importancia de Gran Bretaña y Estados Unidos, 1890-1930
}

\section{Peruvian and Latin American trade relations in the context of changes in the international market: the importance of Great Britain and the United States, 1890-1930}

Franco Lobo Collantes ${ }^{1}$

Universidad del Pacífico

\section{Resumen:}

El presente artículo reconstruye el comercio exterior peruano entre 1890 y 1930 clasificándolo por países, tanto en las importaciones como en las exportaciones por separado, usando las fuentes de origen extranjero de los socios comerciales más importantes: Gran Bretaña y Estados Unidos. El artículo propone que el comercio con Estados Unidos comienza a expandirse desde principios del siglo XX, por lo que el contexto de la Primera Guerra Mundial acelera el proceso. Además, la poca importancia de América Latina como mercado para las manufacturas y proveedor de materia prima para Estados Unidos y Gran Bretaña, desbarata la idea de una relación de dependencia entre la región y las potencias, así como el concepto de imperio informal.

1 Docente en la Universidad del Pacífico. Doctorando en El Colegio de México. Código ORCID: 0000-0002-6277-4904.

Contacto: jllobo@colmex.mx.jf.loboc@up.edu.pe 
Palabras clave: Relaciones comerciales - Exportaciones peruanas - Importaciones peruanas - Gran Bretańa - Estados Unidos

\section{Abstract}

This article reconstructs Peruvian foreign trade between 1890 and 1930, classifying it by country, both in imports and in exports separately, using the sources of foreign origin of the most important trading partners: Great Britain and the United States. The article that proposes trade with the United States begins to expand since the early twentieth century, so the context of the First World War accelerates the process. In addition, the low importance of Latin America as a market for manufactures and raw material supplier for the United States and Great Britain, the idea of a dependency relationship between the region and the powers, as well as the concept of informal empire.

Keywords: Trade relations - Peruvian exports - Peruvian imports - Great Britain - United States

La intensificación de la inserción de América Latina en la economía mundial en el contexto de globalización actual ha despertado el interés de diversos investigadores de las ciencias sociales para comprender esta dinámica y las implicancias que

376 tiene. En el caso de la historiografía latinoamericana, la era exportadora de la segunda mitad del siglo XIX y el primer tercio del siglo XX ha despertado importante interés en los recientes años, ya que nos permite comprender otro proceso similar de rápida inserción en la economía global. Las investigaciones recientes sobre esta era exportadora se han alejado de la visión negativa de la teoría de la dependencia sobre el periodo, y se han centrado en una nueva valoración que tiene como pre- 
misas la reconstrucción empírica y los estudios de caso para inducir conclusiones sobre el proceso en toda la región ${ }^{2}$.

En esta línea historiográfica se enmarca el presente trabajo, el cuál tiene como objetivo analizar las relaciones comerciales de Perú con otros países, con la finalidad de identificar los principales socios comerciales y explicar las implicaciones de ello y sus cambios a lo largo del periodo propuesto. Para ello se han reconstruido las series del comercio exterior del Perú en función de los países con quienes se intercambian los bienes, basados en las principales fuentes externas de las cuales se cuenta: los informes consulares ingleses y la estadística comercial de Estados Unidos. A pesar de ciertos vacíos para algunos años específicos, la fuente nos ha permitido reconstruir con detalle los cambios a largo plazo sobre la mayor o menor participación de algunos países claves y algunas regiones importantes.

Basados en la reconstrucción empírica y la contrastación de la bibliografía existente, el trabajo propone que, a pesar de que la Primera Guerra Mundial fue el periodo clave por el cual Estados Unidos supera Gran Bretaña como el principal socio comercial del Perú, este proceso se observa como una aceleración de una tendencia que apareció desde principios del siglo XX. Además, la participación británica siguió siendo importante en los años veinte, mientras que el mercado latinoamericano (sobre todo en las exportaciones) y los demás países europeos no fueron mercados meramente complementarios, ya que participaron con porcentajes significativos en el comercio peruano. Finalmente, se postula que el mercado latinoamericano y peruano representaron porcentajes poco relevantes para el comercio exterior de las dos potencias del periodo, Gran Bretaña y Estados Unidos, sumándose a la crítica sobre la idea de que

2 Véase en: BULMER-THOMAS, La historia económica; BÉRTOLA Y OCAMPO, El desarrollo económico. Para mayor especificidad sobre el periodo de análisis: KUNTZ, The First Export Era. 
la región era importante como mercado para los productos industriales de estos países y la noción de que América Latina era un proveedor importante de materias primas.

\section{Breve contexto del comercio exterior del Perú}

El arco temporal elegido en el trabajo se sitúa en lo que diversos historiadores económicos denominan primera globalización económica, ya que la integración comercial y la convergencia de precios tuvieron niveles sin precedentes (Williamson, 2012). Esto fue el resultado del impulso generado por la Revolución Industrial, logrando integrar el comercio mundial mediante la reducción de los costes de transporte gracias a las mejoras tecnológicas, sobre todo por la aplicación de la máquina a vapor en el comercio exterior (buques) y en el comercio interno (ferrocarriles) (Findlay y O'Rourke, 2007). Ese contexto de mayor crecimiento de la demanda internacional significó para América Latina una oportunidad para salir del estancamiento que tuvieron la mayoría de países de la región luego del proceso de independencia, sobre todo porque la economía interna no avizoraba alguna posibilidad de salir de esta situación por sí misma (Kuntz, 2017).

En el caso del Perú, antes de la crisis de 1929 la economía experimentó dos auges exportadores. El primero fue el denominado "auge guanero", ya que el Perú tuvo un crecimiento extraordinario de las exportaciones gracias a las ventas del guano, fertilizante natural que fue muy demandado en la agricultura

378 europea y estadounidense en la segunda mitad del siglo XIX. Este auge exportador se interrumpió estrepitosamente con la Guerra del Pacífico (1879-1883), conflicto que enfrentó a los países de Chile, Bolivia y Perú, con consecuencias devastadoras para la economía peruana, ya que fue despojada de las principales exportaciones (guano y salitre) y cargó con una enorme deuda externa producto de la inversión en ferrocarriles de la década de 1870. La década de 1880 fue dura para la 
economía peruana, pero a partir de 1890 la situación cambio gracias básicamente a tres acontecimientos. Primero, la firma del Contrato Grace en 1889, que significó el arreglo de la deuda externa y la reinserción en el mercado mundial de capitales. Segundo, la estabilidad política que se consiguió luego de la última crisis de interna de 1894-1895, producto de la guerra civil que enfrentó el caudillo Nicolás de Piérola contra el gobierno de Andrés Avelino Cáceres (Thorp y Bertram, 2013). Tercero, la reforma fiscal que se implementó para incrementar la recaudación del estado tuvo niveles bastante bajos para las exportaciones, lo que incentivó su crecimiento y el crecimiento de la élite local (Contreras, 2012).

Gracias a las nuevas circunstancias más favorables, el Perú experimentó el segundo auge exportador de su historia republicana, el cuál duro hasta 1930. Este segundo auge tuvo algunas características particulares. En primer lugar, la élite local cumplió un papel fundamental, ya que lideró el proceso de recuperación de las exportaciones dado la poca inversión extranjera en el país ante el fuerte endeudamiento. A pesar de la fuerte presencia inglesa y estadounidense en algunos sectores estratégicos durante nuestro periodo de análisis, la élite preservó su mayoritaria participación en la exportación agrícola en la costa (Thorp y Bertram, 2013). De hecho, el contacto de los grupos económicos nativos con las grandes empresas extranjeras generó un aprendizaje para las empresas peruanas en términos de estrategias comerciales, lo que les permitió defender sus intereses (Quiroz, 1990). En segundo lugar, la canasta de exportación fue bastante diversificada, donde el mayor porcentaje hasta 1910 fue la de productos agrícolas como el azúcar y el algodón, para luego pasar al liderazgo de cobre y petróleo que se afianzaron durante la Primera Guerra Mundial. En los años veinte la distribución porcentual fue más pareja entre varios productos, aunque tuvo la mayor participación el petróleo (Contreras, 2011). Esta diversidad convirtió al Perú en el país con menor concentración de productos de exporta- 
ción en América Latina antes de la Primera Guerra Mundial haciéndolo menos dependiente y, por ende, menos vulnerable a los choques externos (Bulmer-Thomas, 2010). En tercer lugar, la contribución de las exportaciones al crecimiento del PIB del país fue significativo, ya que osciló entre $15 \%$ y $22 \%$ (Zegarra, 2017). Este crecimiento le permitió incrementar su poder de compra, el cual tuvo un crecimiento promedio en el periodo de $4.5 \%$, convirtiéndose en el segundo país latinoamericano en crecimiento del poder de compra entre 1890 y 1912 (Zegarra, 2017; Bulmer-Thomas, 2010). Esta capacidad de compra le dio la posibilidad de incrementar las importaciones, dinámica que mostramos en el Gráfico 1. En él se deja notar el crecimiento constante de las exportaciones, y cómo las importaciones se rezagan desde el contexto de la Primera Guerra Mundial al perder socios comerciales europeos clave.

\section{Gráfico 1. Desempeńo del comercio exterior del Perú, 1890 y 1930}

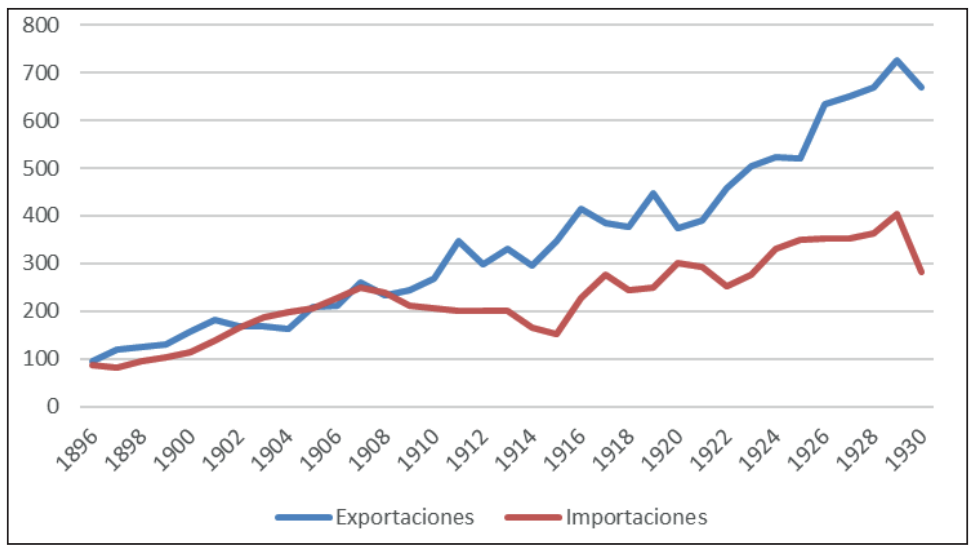

Elaboración propia a partir de datos de: Seminario, Bruno, La economía peruana en la era moderna, Lima, Universidad del Pacífico, 2015.

Con este panorama general del periodo podemos situarnos mejor para comprender los contextos de cambios entorno a la participación porcentual de las potencias económicas de la época en el comercio exterior peruano. 


\section{Los socios comerciales del Perú}

Para explicar la dinámica a largo plazo de los socios comerciales del Perú entre 1890 y 1930, se ha decidido iniciar desde la evidencia empírica encontrada, a partir de la cual desarrollaremos la explicación. El Gráfico 2 y 3 muestran la composición porcentual de las importaciones y exportaciones en el Perú, respectivamente, según los países de destino para nuestro periodo de análisis ${ }^{3}$. Tanto en las exportaciones como en las importaciones, la década de 1890 inició con la mayor participación de Gran Bretaña, una participación secundaria de Estados Unidos y otra participación notable de otras regiones. En el caso de la región Latinoamericana, la principal porción del comercio correspondió al pacífico sudamericano, sobre todo con Chile, país que fue uno de los principales mercados de exportación peruana. En el caso del resto de Europa, el país con mayor participación fue Alemania, y en menor medida Francia y Bélgica.

Gráfico 2. Composición de las importaciones peruanas según país de destino, 1891-1930

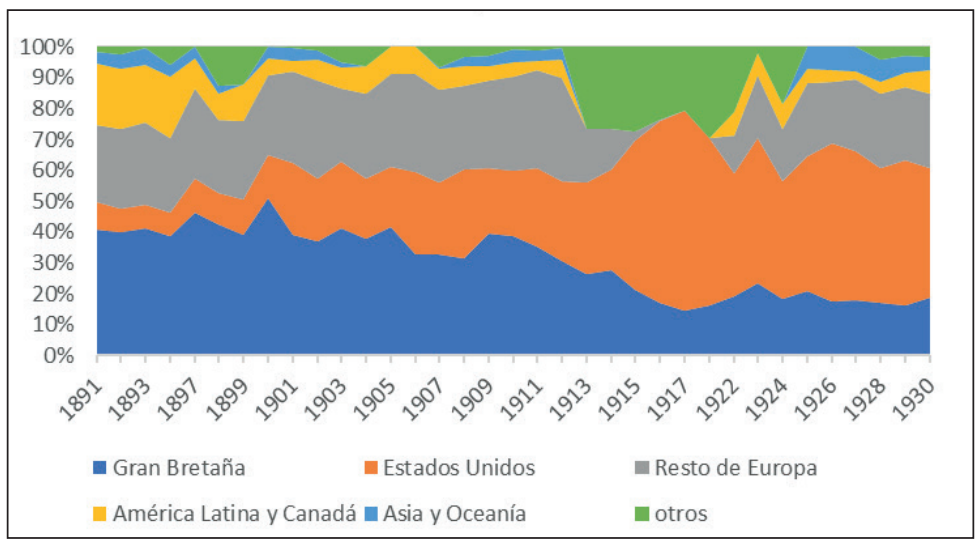

3 Gráficos basados en los anexos 1 y 2. 
Gráfico 3. Composición de las exportaciones peruanas según país de destino, 1891-1930

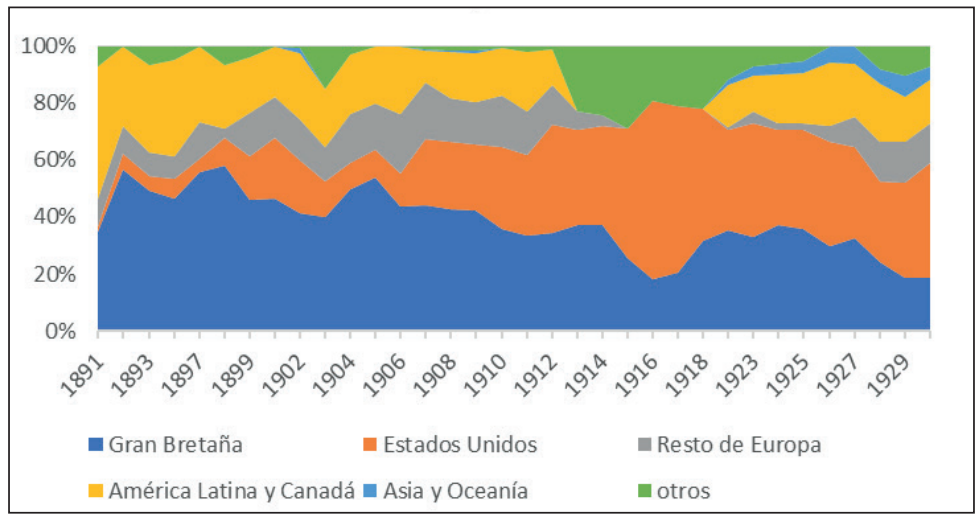

La mayor participación de Gran Bretaña no sorprende, dado su potencial industrial, comercial y, sobre todo, el liderazgo en los flujos de capital antes de la Primera Guerra Mundial. En 1899, Gran Bretaña fue el país con mayor participación en las exportaciones de manufacturas en el mundo con 33\%, mientras que en 1913 fue el país con mayor inversión extranjera del globo, representando el $50 \%$ en exportación de capitales (Yepes, 1972). En el caso latinoamericano, el compromiso de la región con el crecimiento liderado por las exportaciones y el libre tránsito de capitales fue muy compatible con las relaciones económicas de Gran Bretaña con el mundo, lo que aceleró el proceso de integración económica. Esto se puso en evidencia con el aumento del comercio exterior, ya que la participación de las exportaciones británicas en América Latina creció entre 1860 y 1913 de 7\% a $11 \%$, mientras que en el caso en las importaciones el aumento fue de $6 \%$ a $10 \%$ en el mismo periodo (Boyns y Gray, 2016). Sin embargo, la relación comercial entre la región y la potencia británica fue mucho más estrecha en términos de inversión y flujos de capital. De hecho, el país que recibió mayor inversión británica, Argentina, tuvo niveles cer- 
canos a los recibidos por colonias británicas como la India y Ceilán (Yepes, 1972). En términos generales, Gran Bretaña representó el $68 \%$ de la deuda pública latinoamericana en 1914, y en inversión extranjera directa, participó con 47\%. (Bulmer-Thomas, 2010). Las inversiones británicas en la región tuvieron una amplia diversificación en varios sectores productivos, siendo uno de los principales la construcción de ferrocarriles, lo que permitió en buena medida la expansión ferroviaria latinoamericana entre 1850 y 1914 (Miller, 1995; Markaham, 2017).

En el Perú, la mayor participación de Gran Bretaña en el comercio exterior se evidencia en la reconstrucción de nuestras cifras que se observa en el Gráfico 2 y 3 . En el caso de las exportaciones, entre 1892 y 1909 Gran Bretańa tuvo una participación entre $40 \%$ y $50 \%$, mientras que, en las importaciones, entre 1891 y 1911 tuvo una participación entre $30 \%$ y $45 \%$. Esta relevante presencia británica en el comercio exterior peruano tiene importantes antecedentes en el auge guanero, ya que el principal mercado de destino fue Gran Bretaña y la distribución del producto estuvo mayoritariamente en manos de casas comerciales inglesas (Miller y Greengil, 2017). Como mencionamos en la contextualización, la fuerte crisis de la deuda peruana luego de la Guerra del Pacífico se solucionó gracias al Contrato Grace. La mayor parte de los tenedores de la deuda fue británica, por lo que el Contrato Grace implicó un arreglo con el capital británico, de manera que se suspendió la deuda externa peruana a cambio de una serie de concesiones a dichos tenedores. Las concesiones más importantes fueron la entrega de la administración de todos los ferrocarriles del Perú por 66 años, dos millones de hectáreas en la Amazonía, dos millones de toneladas de guano y una cuota anual de 80 mil libras esterlinas durante 33 ańos (Contreras y Cueto, 2009). Para administrar estas concesiones, los tenedores de la deuda formaron la compañía Peruvian Corporation, la que se convirtió en la 
principal inversión extranjera en el Perú de finales del siglo XIX y principios del siglo XX. A pesar de que la posesión de los ferrocarriles no les generó amplios réditos, su diversificación en diversas áreas, como la producción y exportación de petróleo, le dio una posición sólida en la economía peruana hasta 1910 (Miller, 2011). Además de esta inversión, la presencia de casas comerciales fue significativa durante todo el periodo de análisis. Estas casas comerciales tuvieron fuerte presencia en el proceso de recuperación económica luego del conflicto con Chile, siendo claves como agentes de comercialización de los principales productos agrícolas de exportación del Perú, así como los más relevantes importadores de productos europeos para el mercado peruano. Adicionalmente, estas casas comerciales diversificaron sus intereses en distintas actividades productivas en el Perú, realizando integraciones en los sectores con mayor experiencia. El caso de la empresa Duncan Fox ejemplifica lo mencionado, ya pasó de ser uno de los principales agentes en la exportación de algodón, a ser uno de los principales productores de este, además de convertirse en una de las empresas más importantes en la producción de textiles de algodón para el mercado interno (Miller, 2011; Monsalve, 2011). Cabe destacar que, durante el periodo de análisis, las importaciones iniciaron una fuerte diversificación, ya que al mayoritario rubro de textiles se le añadió importantes cantidades de otros bienes semi-procesados, bienes de capital y herramientas (Bonilla, 1980).

Sin embargo, esta mayor participación inglesa en el comercio exterior fue gradualmente desafiada por otros socios comerciales, ente los cuales Estados Unidos se convirtió en la principal competencia en el mercado latinoamericano. Lo que muestra nuestros gráficos es que el crecimiento de la participación de Estados Unidos en el comercio exterior del Perú fue constante desde 1890, pero recién entre 1905 y 1910 comenzó a restar participación a Gran Bretaña. Por ello, la Primera Guerra Mundial es el proceso de aceleración de la 
presencia estadounidense en el comercio exterior peruano, lo que implicó reemplazar a Gran Bretaña en la participación mayoritaria, hecho que se mantiene en los ańos veinte.

El crecimiento del comercio exterior de Estados Unidos en el Perú respondió a una lógica global, ya que entre 1899 y 1913 el gigante norteamericano incrementó su participación mundial en las exportaciones de manufacturas de $11.7 \%$ a $13 \%$, respectivamente. Además, cabe destacar la importancia del mercado europeo, sobre todo en el caso específico de Alemania, el principal socio comercial del Perú durante nuestro periodo de análisis, luego de Gran Bretańa y Estados Unidos. Debido al crecimiento industrial experimentado en la segunda mitad del siglo XIX, Alemania incrementó su participación en las exportaciones de manufacturas en el mundo de $22.4 \%$ en 1899 a $26.6 \%$ en 1913 . Sumado a ello, en 1914 era el tercer país con la mayor participación en exportaciones de capital en el globo con 7.3\%. De hecho, América Latina significó alrededor de la cuarta parte de la inversión alemana en el mundo, sobre todo por sus inversiones en Argentina (Yepes, 1972).

Volviendo al eje de Estados Unidos, las inversiones en América Latina representaron el 50\% de las inversiones estadounidenses en el mundo para 1913, aunque estaba aún por debajo de la cantidad invertida en la región por Gran Bretaña. Sin embargo, la Primera Guerra Mundial implicó un retroceso de los capitales europeo, vacío que fue cubierto por la expansión norteamericana, ya que duplicó sus inversiones en Latinoamérica entre 1914 y 1919. Durante los primeros treinta ańos del siglo XX, esta inversión estuvo dirigida a los sectores de exportación más rentable, como la minería, el petróleo y la agricultura, y adicionalmente a la expansión de servicios públicos (Yepes, 1972; Bulmer-Thomas, 2010; Bértola y Ocampo, 2013). Sin embargo, en términos del comercio exterior, antes de iniciar la Primera Guerra Mundial, Estados 
Unidos se había convertido en el principal socio comercial de América Latina, desplazando a Gran Bretaña, proceso que se aceleró con la Primera Guerra Mundial (Bulmer-Thomas, 2010).

Con respecto al Perú, el acercamiento con Estados Unidos en el periodo que analizamos tiene tres hitos. El primero fue el propio Contrato Grace que ya hemos comentado, ya que lleva el nombre de la que se convertirá en la casa comercial extranjera más importante en el Perú del primer tercio del siglo XX. De hecho, fue Michael Grace el verdadero beneficiado del contrato que lleva su apellido, ya que obtuvo una participación importante como accionista en las principales empresas del país. Por el contrario, el desempeño de los ferrocarriles en manos de la empresa inglesa evidenció pocos réditos en el corto y largo plazo, así como los pocos efectos en la economía peruana (Miller, 2011). La importancia de la Grace en promover la inversión estadounidense en diversos sectores de la economía peruana y el aumento de comercio entre ambos países fue impulsada gracias al propio proceso de crecimiento y modernización de las dos economías, con mayor intensificación en el caso norteamericano (Clayton, 1998). Debido a este proceso de acercamiento, las inversiones directas estadounidenses en Perú se incrementaron de 6 millones de dólares a 63 millones, entre 1897 y 1914, respectivamente (Yepes, 1972). En términos de comercio exterior, gracias a la reconstrucción que hemos hecho para el caso peruano, hacia 1913 Estados Unidos aún no superaba por completo a Gran Bretańa como en el promedio Latinoamericano, pero sí tuvo un crecimiento notable. Entre 1891 y 1913, la participación de Estados Unidos en las importaciones peruanas pasó de $7.8 \%$ a $29.8 \%$, superando a Gran Bretańa, mientras que, en el caso de las exportaciones, su participación pasó de $1.6 \%$ a $33.2 \%$, que a pesar de significar un mayor crecimiento que las importaciones, no pudo superar a Gran Bretańa, aunque estuvo muy cerca. 
El segundo hito se refiere a la Primera Guerra Mundial, proceso alineado con el contexto latinoamericano que hemos explicado, lo que implicó en términos comerciales convertir a Estados Unidos en el primer socio comercial del país. Para 1918, Estados Unidos participaba con $54.3 \%$ en las importaciones del Perú, mientras que en las exportaciones participó con 46.5\%. El tercer hito fue la llegada al gobierno de Augusto Leguía, presidente del Perú entre 1919 y 1930, cuando el acercamiento entre ambos países llegó a su máxima expresión. La intención de acercar al Perú con Estados se observa en el nombramiento de especialistas, administradores y oficiales navales estadounidenses en varios cargos importantes de la administración pública, como el Ministerio de Educación, la Aduana o el Banco de Reserva (Clayton, 1998).

En este acercamiento también cumplieron un rol importante las empresas extranjeras. Fueron diversas las empresas estadounidenses establecidas en el Perú (Burga y Flores Galindo, 1987; Clayton, 1998), sin embargo, destacaron tres: la Cerro de Pasco Mining Corporation, la International Petroelum Company (IPC) y la ya mencionada W.R. Grace. A principios del siglo XX se estableció la Cerro de Pasco Corporation, adquiriendo las minas en la región del mismo nombre, en la sierra central del Perú, donde la inversión clave fue la terminación de la línea férrea entre Cerro de Pasco y La Oroya, lo que permitió unir el centro minero con el puerto del Callao. Con el tiempo adquirió otras inversiones mineras importantes, lo que probablemente significó la mayor inversión minera en cobre en todo el mundo y, ciertamente la más grande de Sudamérica en 1916. Para la década de 1920 la empresa amplió sus inversiones en minas de carbón, sal y otros minerales, demostrando una gran capacidad de diversificación. En el caso de la casa comercial W.R. Grace, tuvo una presencia importante desde el siglo XIX en la comercialización del guano, y gracias al Contrato Grace, amplió sus 
inversiones en el Perú para inicios del siglo XX. En la década de 1920, dominó el transporte marítimo entre Perú y Estados Unidos, eran los segundos productores de azúcar en el país, administraban las principales fábricas textiles e incluso fueron pioneros en el transporte aéreo internacional. Finalmente, la IPC llegó al Perú más tardíamente, en 1913, al adquirir la London and Pacífic y otras pequeñas empresas de producción y comercialización de petróleo, convirtiéndose en la principal productora de petróleo del país, sólo compartiendo una porción menor del mercado con la británica Lobitos. La inversión llegó por parte de la expansión de la Standard Oil of New Jersey, una de las corporaciones independientes creadas como consecuencia del desmembramiento de la Standard Oil Company. Sin embargo, debido a los conflictos con el gobierno peruano, la empresa recién expandió significativamente su producción y exportación en la década de 1920, gracias a la mejor predisposición del gobierno de Augusto Leguía. De las tres compañías mencionadas, la IPC fue la que menos se diversificó a otros sectores, ya que la inversión se limitó al propio sector, como la refinería de Talara, la construcción del puerto de la misma localidad y la construcción de casas para los empleados de la compañía (Clayton, 1998). Esto ha llevado a algunos autores a considerar esta inversión como un tipo de enclave, debido al poco valor de retorno que generó la empresa (Thorp y Bertram, 2013; Clayton, 1998). Sin embargo, este tipo de valoraciones carecen de comparar el ingreso generado por la actividad exportadora con la economía peruana, ya que, por más que el porcentaje de retorno sea muy pequeño con respecto a lo que se enviaba al exterior, si ese porcentaje que se queda en el mercado interno significa un volumen muy grande frente a la economía local, el impacto es más importante de lo que se imagina (Kuntz, 2017).

Como hemos visto, los años veinte significó el afianzamiento de Estados Unidos como principal socio comercial y pro- 
veedor de capital en América Latina, lo que no implicó una desaparición de la influencia británica. En el Perú, si bien en 1930 alrededor del 40\% del comercio exterior era con Estados Unidos y las inversiones estadounidenses en Perú bordeaban los 200 millones de dólares, Gran Bretaña aún participaba con $20 \%$ en el comercio exterior peruano en el mismo año, y en 1925 tenía invertido 125 millones de dólares en el Perú (Clayton, 1998). De hecho, para 1928, la inversión británica en Argentina, Brasil y México era muy importante (Vargas, 2000). Esta presencia británica puede responder a una especie de reflote en la región luego del alejamiento en tiempos de guerra, un reflote basado en los fuertes lazos económicos con la región durante buena parte del siglo XIX. Esto cobra sentido cuando se observa la preocupación por parte de las autoridades del comercio exterior británico por recuperar en la posguerra el espacio perdido durante el conflicto (Markhan, 2017). Sin embargo, la posible rivalidad angloamericana no ha tenido la atención necesaria por parte de la historiografía, salvo casos concretos (Vargas, 2000). Desde este punto de vista, cabría añadir que mayoría de las ganancias generadas en la Guerra para Estados Unidos fueron invertidas en su economía interna. Además, a pesar del liderazgo norteamericano, aún no tenía las habilidades necesarias en términos de relaciones internacionales que sí tuvo Gran Bretaña gracias a la experiencia ganada durante el siglo XIX (Kennedy, 1980). Este aprendizaje probablemente lo haya desarrollado Estados Unidos durante las guerras mundiales y puesto en práctica en la contienda de la Guerra Fría. Sin embargo, para nuestro periodo de análisis, los estudios para el caso latinoamericano aún son limitados.

\section{¿Somos tan importantes?}

La presencia inglesa y norteamericana generaron diversas críticas en América Latina desde las décadas de 1930 y 1940 con el surgimiento de gobiernos nacionalistas en contextos 
de crisis internacional (Miller, 1995). Posteriormente, una vertiente del marxismo latinoamericano estructuró una visión negativa sobre la dinámica económica que relacionaba a los países de la región con el mundo desarrollado, la denominada teoría de la dependencia, con gran apogeo entre las décadas de 1960 y 1990. Potenciados posteriormente por las teorías del sistema mundo de Inmanuelle Wallesrstein, este enfoque argumentaba que la inserción latinoamericana en el mercado mundial había sido condicionada a la dinámica económica de los países desarrollados, lo que implicó una inserción subordinada o dependiente. De esta manera, la lógica de la división mundial del trabajo generó que la región fuera exportadora de las materias primas necesarias para el desarrollo industrial de los países más ricos, y a la vez era un importante mercado para los bienes manufactureros de estos países. Por el lado de los estudios anglosajones, en los años cincuenta apareció el concepto de "Imperio Informal", el cuál tuvo una gran acogida en la comunidad académica. John Gallager y Ronald Robinson acuńaron el concepto para explicar la relación de Gran Bretańa con América Latina en el siglo XIX, la cuál no podía ser separada de la expansión imperial británica. El argumento central fue que las élites de América Latina habían interiorizado la ideología del libre comercio de tal manera que hizo innecesaria una intervención política por parte de Gran bretaña. La colaboración informal de las élites latinoamericanas con los empresarios extranjeros sirvió a los intereses británicos casi tan bien como al imperio formal, aunque mucho menos costoso (Miller, 1995).

Tanto la teoría de la dependencia como el concepto de imperio informal influenciaron en la historiografía peruana con trabajos que tuvieron amplia aceptación en la comunidad académica del país. De hecho, para estos autores, el cambio de siglo significó pasar de la hegemonía británica a la hegemonía estadounidense, pero manteniendo un papel subordinando de periferia proveedora de materia prima y receptora 
de bienes industriales ${ }^{4}$. Sin embargo, desde finales de la década de 1970 diversos estudios en América Latina fueron cuestionando con evidencia empírica los supuestos de la teoría de la dependencia, ya que reducían la importancia de América Latina para Gran Bretaña y, en menor medida, para Estados Unidos. Desde el lado anglosajón, los trabajos de Platt desde finales de la década de 1960 demostraron que el gobierno británico no siempre estuvo interesado y menos involucrado en la región, criticando la idea de una influencia externa directa en la política de los países latinoamericanos (Miller, 1995). Recientemente en Perú también estas tendencias revisionistas han erosionado las posturas emparentas con la teoría de la dependencia y el imperio informal, demostrando que las grandes corporaciones extranjeras ingresaron y se desempeñaron en términos básicamente económicos, con gran independencia del gobierno peruano y una élite nativa que buscó defender sus intereses constantemente (Miller, 2011).

En línea con lo mencionado, actualmente es bien conocido la poca importancia que tuvieron los países llamados periféricos como proveedores de materias primas a los países industriales durante el siglo XIX y las primeras décadas del siglo XX, así como la minúscula cuota de mercado que significó la región para las manufacturas del mundo desarrollado. Por ejemplo, el principal exportador de carbón en el siglo XIX, la fuente de energía clave para el desarrollo económico del siglo, fue Gran Bretaña, mientras que la otra potencia económica de principios del siglo XX, Estados Unidos, era el primer productor y exportador de petróleo. En suma, la autosuficiencia energética de los países desarrollado fue evidente durante nuestro periodo de análisis. Otro caso significativo es el hierro, el metal más importante en el comercio de minerales en el contexto de la primera globalización. El 98\% del consumo

4 Véase en: BONILLA, Gran Bretaña y el Perú. BURGA Y FLORES, Apogeo y crisis. YEPES, Perú, 1820-1920. BONILLA, Un siglo a la deriva. 
de este metal en los países desarrollados fue producido en esta zona, y sólo el $2 \%$ fue importado desde el tercer mundo. En términos generales, se estima que la autosuficiencia de los países desarrollados entre 1800 y 1913 fue entre 96\% y 102\% (implica margen de exportación) (Bairoch, 1995). Por lo tanto, la idea de que el mundo no industrial preveía de materias primas necesarias para las industrias en los países desarrollados quedaría sin sentido.

Para sumarnos al debate, las fuentes analizadas nos han permitido reconstruir la participación latinoamericana y peruana en las importaciones de los dos países más importantes del nuestro periodo, Estados Unidos y Gran Bretaña. El Gráfico 4 nos muestra que la participación Latinoamericana en Estados Unidos varía entre $20 \%$ y $35 \%$, obteniendo una cuota significativa, pero en el caso británico esta no supera el $10 \%$. El gráfico además muestra la mínima participación peruana en la cuota latinoamericana, no superando el $5 \%$.

La otra suposición importante sobre la relación comercial entre las regiones de la periferia primario-exportadora y los centros industriales, es que los primeros sirven de mercados para las manufacturas de los segundos. Sin embargo, la evidencia empírica es contundente: sólo el 17\% de la exportación del mundo desarrollado fue hacia el tercer mundo entre 1800 y 1930. En el caso específico de las manufacturas, la participación del tercer mundo varió entre $6 \%$ y $14 \%$, lo que reduce su importancia como potencial mercado consumidor (Bairoch, 1995).

En el Gráfico 6 se observa que la participación de América Latina en ambas potencias es pareja hasta 1910. Entre 1915 y 1930, como efectos de la Primera Guerra Mundial, el mer-

5 No se encontraron datos para la partición peruana en el mercado británico después de 1910. 
cado latinoamericano eleva su cuota del mercado en Estados Unidos hasta un poco más de 18\%, mientras que Gran bretaña reduce la participación de la región en sus exportaciones hasta alrededor del 7\% en la década de 1920. En el caso peruano, su participación en América Latina es pareja para Estados Unidos y Gran Bretaña desde 1900. Lamentablemente, la falta de datos para Gran Bretańa evita que podamos analizar la participación de Perú en la región entre 1910 y 1930. En general, este Gráfico muestra la dinámica del impacto de la Primera Guerra Mundial y el mayor acercamiento con Estados unidos que explicamos en el primer apartado. En suma, ambos gráficos presentados refuerzan la idea de reducir la importancia de América Latina en el comercio exterior con los países potencias del periodo analizado, aunque es evidente que el acercamiento con Estados Unidos es mayor que con Gran Bretaña. En ese marco, Perú es un mercado bastante marginal, ya que su participación dentro de Latinoamérica en esta dinámica osciló entre 1\% y 12\%.

Gráfico 5. Participación porcentual de América Latina y Perú en las importaciones de Estados Unidos y Gran Bretańa, 1890-1930

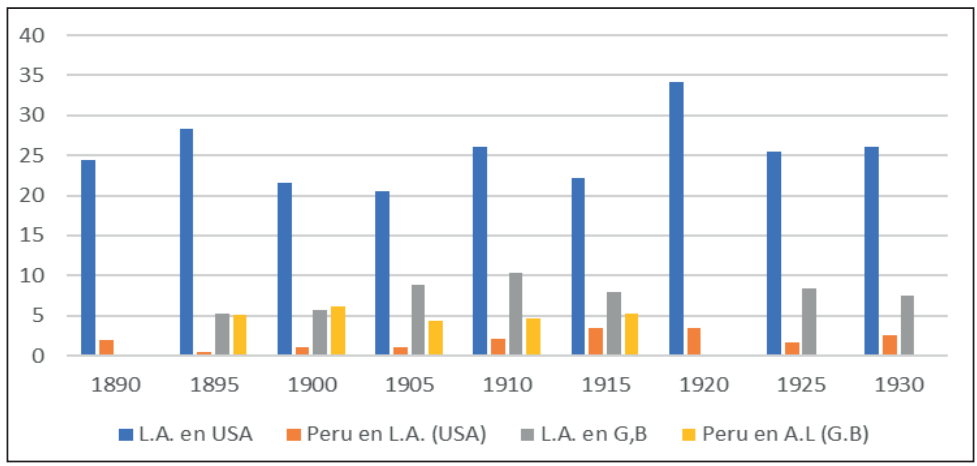


Gráfico 6. Participación porcentual de América Latina y Perú en las exportaciones de Estados Unidos y Gran Bretańa, 18901930

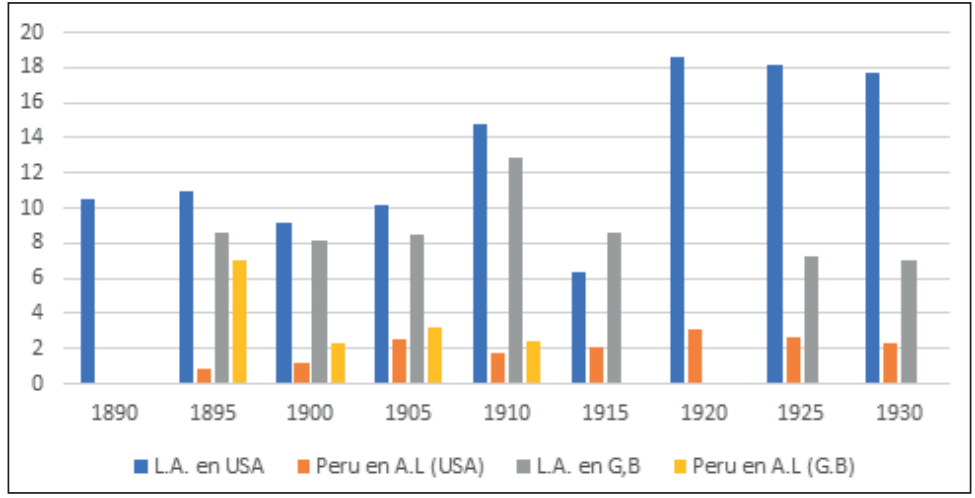

\section{Conclusiones}

La reconstrucción de la participación porcentual de los países con los que Perú comerció entre 1890 y 1930 nos ha permitido sumarles nuevas aristas a los procesos conocidos en América Latina. Además del evidente tránsito que significó la Primera Guerra Mundial de una mayoritaria participación británica a una estadounidense, nuestras cifras evidencian que el crecimiento de Estados Unidos desde finales del siglo XIX comenzó a reducir la cuota británica entre 1905 y 1910, por lo que la transformación de la Gran Guerra fue una aceleración de un proceso anterior. Además, a pesar de que este trabajo se centro en explicar la relación del Perú con estos dos principales socios comerciales, la participación de Chile y el mercado latinoamericano, así como Alemania y el mercado europeo, no cumplieron un rol marginal, participando con un promedio entre $20 \%$ y $30 \%$ entre ambos mercados, lo que evidencia una gran diversificación de los socios comerciales. Ahora bien, ¿el hecho de tener mayor participación en el comercio exterior con el Perú implicó control hegemónico de parte de 
Gran Bretaña o Estados Unidos? Este razonamiento propio de la teoría de la dependencia ha sido más teórico que empírico, ya que abundante literatura en la región ha demostrado que esa mayor participación respondió a que dichos países eran los mercados más importantes del periodo, por lo que tener una mayor conexión con esos mercados es casi una consecuencia natural del proceso de integración económica global.

Adicionalmente, las fuentes nos permitieron reconstruir la participación comercial de América Latina y Perú en el comercio exterior de Estados Unidos y Gran Bretaña. Los resultados arrojan una pequeña participación de la región en ambos países (con mayores porcentajes en el caso estadounidense), y una posición completamente marginal del mercado peruano en esta lógica. Estos resultados se suman a la posición revisionista que ha cuestionado seriamente la idea que América Latina, como parte del tercer mundo o la periferia, fue importante proveedora de materias primas para el mundo desarrollado, así como un mercado importante de consumo de manufacturas. Los alcances de los principios o supuestos de la teoría de la dependencia y del concepto de imperio informal cada vez se hacen menos aplicables.

Recibido: 15 de mayo de 2020

Aprobado: 25 de setiembre de 2020

\section{Referencias bibliográficas}

BAIROCH, P.

(1995) Economics and World History. Myths and Paradoxes. Chicago: University of Chicago Press.

BÉRTOLA, L. y OCAMPO, J.

(2013) El desarrollo económico de América Latina desde la independencia. México D.F.: Fondo de Cultura Económica. 
BONILLA, H.

(1975) Gran Bretaña y el Perú. Lima: Instituto de Estudios Peruanos.

BONILLA, H.

(1980) Un siglo a la deriva. Ensayos sobre el Perú, Bolivia y la guerra. Lima: Instituto de Estudios Peruanos.

BOYNS, T. y GRAY, S.

(2016) Welsh coal and the informal empire in South America, 1850-1913. Atlantic Studies, 13 (1), 53-77. https://doi.org/10.1080/14788810.2015.1110678

BULMER-THOMAS, V.

(2010) La historia económica de América Latina desde la Independencia. México: Fondo de Cultura Económica.

BURGA, M. y FLORES GALINDO, A.

(1987) Apogeo y crisis de la república aristocrática. Lima: Rikchay Perú.

CLAYTON, L.

(1998) Estados Unidos y el Perú 1800-1995. Lima: Centro de Estudios Internacionales.

CONTRERAS, C. y CUETO, M.

(2009) Historia del Perú contemporáneo. Lima: Instituto de Estudios Peruanos.

396 (2011) Economía de la primera centuria independiente. Lima: Instituto de Estudios Peruanos, Banco Central de Reserva del Perú.

CONTRERAS, C.

(2012) La economía pública en el Perú después del guano y del salitre. Crisis fiscal y élites económicas durante su primer siglo independiente. Lima: Instituto de Estudios Peruanos, Banco Central de Reserva del Perú. 
FINDLAY, R. y O'ROURKE, K.

(2007) Power and Plenty. Trade, War, and the World Economy in the Second Millennium. New Jersey, Princeton University Press. https://doi.org/10.1515/9781400831883

KENNEDY, D.

(1980)

Over Here. The First World War and American Society. New York, Oxford University Press.

KUNTZ, S.

The First Export Era. Reassessing its Contribution to Latin American Economies. London: Palgrave Studies in Economic History. https://doi. org/10.1515/9781400831883

MARKHAM, B.

(2017) The Challenge to 'Informal' Empire: Argentina, Chile and British Policy-Markers in the Inmediate Aftermath of the First World War. The Journal of Imperial and Commonwealth History, 45 (3), 449-474. https://doi.org/10.1080/03086534.2017.1332132

MILLER, R.

British Investment in Latin America, 1850-1950 A Reappraisal, Journal of Iberian and Latin American Economic History, 19 (3), 21-52. https://doi. org/10.1017/S0165115300021318

MILLER, R.

(2011) Empresas Británicas, economía y politica en el Perú, 1850-1934. Lima: Instituto de Estudios Peruanos, Banco Central de Reserva del Perú.

MILLER, R. y GREENHILL, R.

(2017) Las cadenas internacionales de dos fertilizantes: guano y nitrato, de 1840 a 1930. En: Marichal, C., Topik, S. y Frank, Z. (Eds), De la plata a la cocaina. Cinco siglos de historia económica de América Latina, 1500-2000 (321-381). México D.F.: Fondo de Cultura Económica. 
MONSALVE, $M$.

(2011) Industria y mercado interno, 1821-1930. En: Contreras C. (Ed.), La economía peruana en su primera centuria. (239-301). Lima: 2011.

QUIROZ, A.

(1990) Banqueros en conflicto: estructura financiera y economía peruana, 1884-1930. Lima: Centro de Investigación de la Universidad del Pacífico.

SEMINARIO, B.

(2015) El desarrollo de la economía peruana en la era moderna. Precios, población, demanda y producción desde 1700. Lima: Universidad del Pacífico.

THORP, R. y BERTRAM, G.

(2013) Perú, 1890-1977: crecimiento y politicas en una economía abierta. Lima: Universidad del Pacífico.

VARGAS, E.

(2000) Anglo-American Rivalry in Brazil: the Case of the 1920s. Oxford: Centre for Brazilian Studies, University of Oxford.

WILLIAMSON, J.

(2012) Comercio y pobreza. Cuando y cómo comenzó el atraso del Tercer Mundo. Barcelona, Crítica.

YEPES, E.

(1972) Perú, 1820-1920: un siglo de desarrollo capitalista. Lima: Instituto de Estudios Peruanos.

398 ZEGARRA, L.

(2017) Exports and Their Impact on the Economy. The Case of Peru, 1830-1930. En Kuntz, S. (ed), The First Export Era. Reassessing its Contribution to Latin American Economies (279-312). London: Palgrave Studies in Economic History. https://doi.org/10.1007/9783-319-62340-5_8 


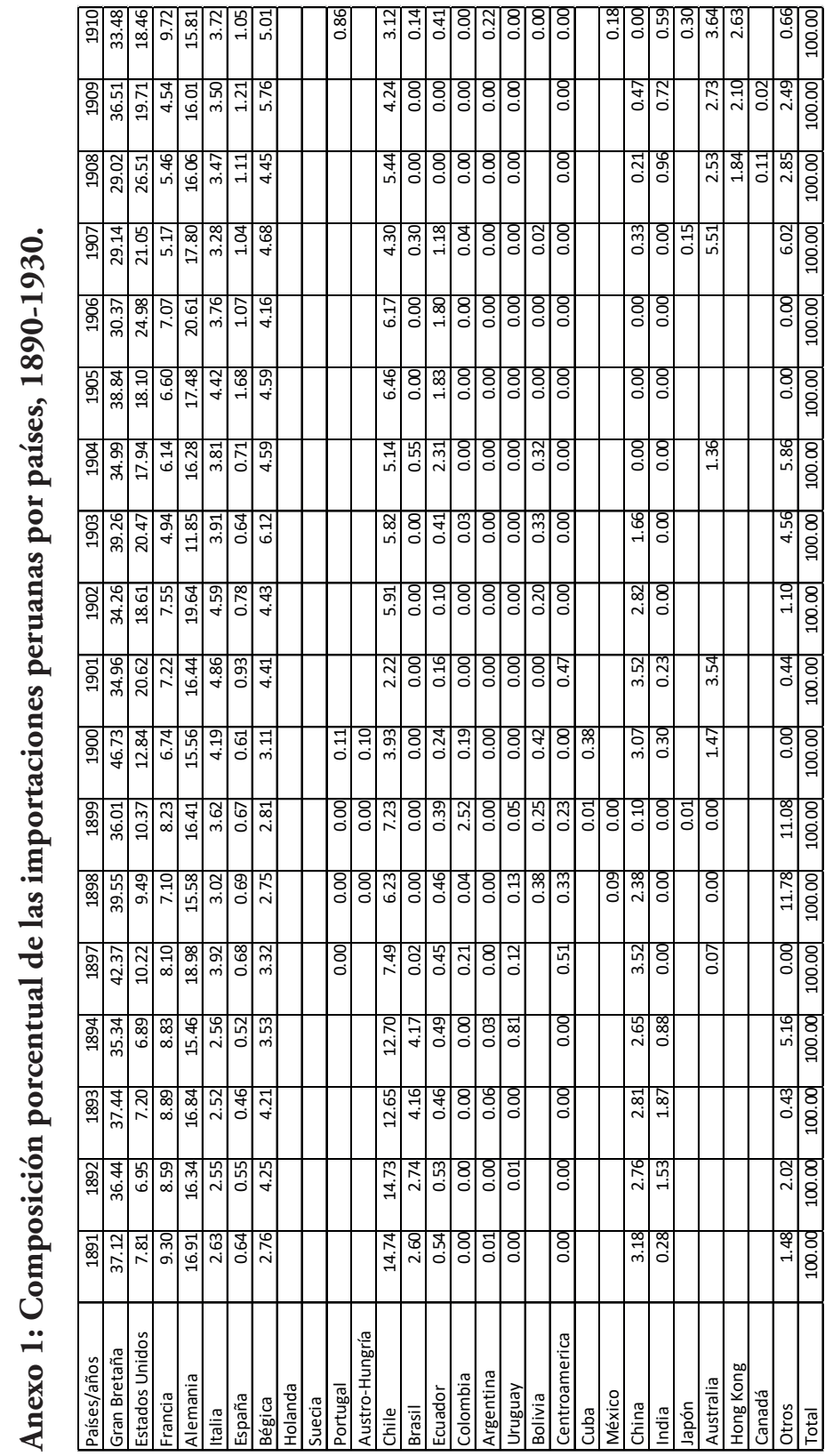




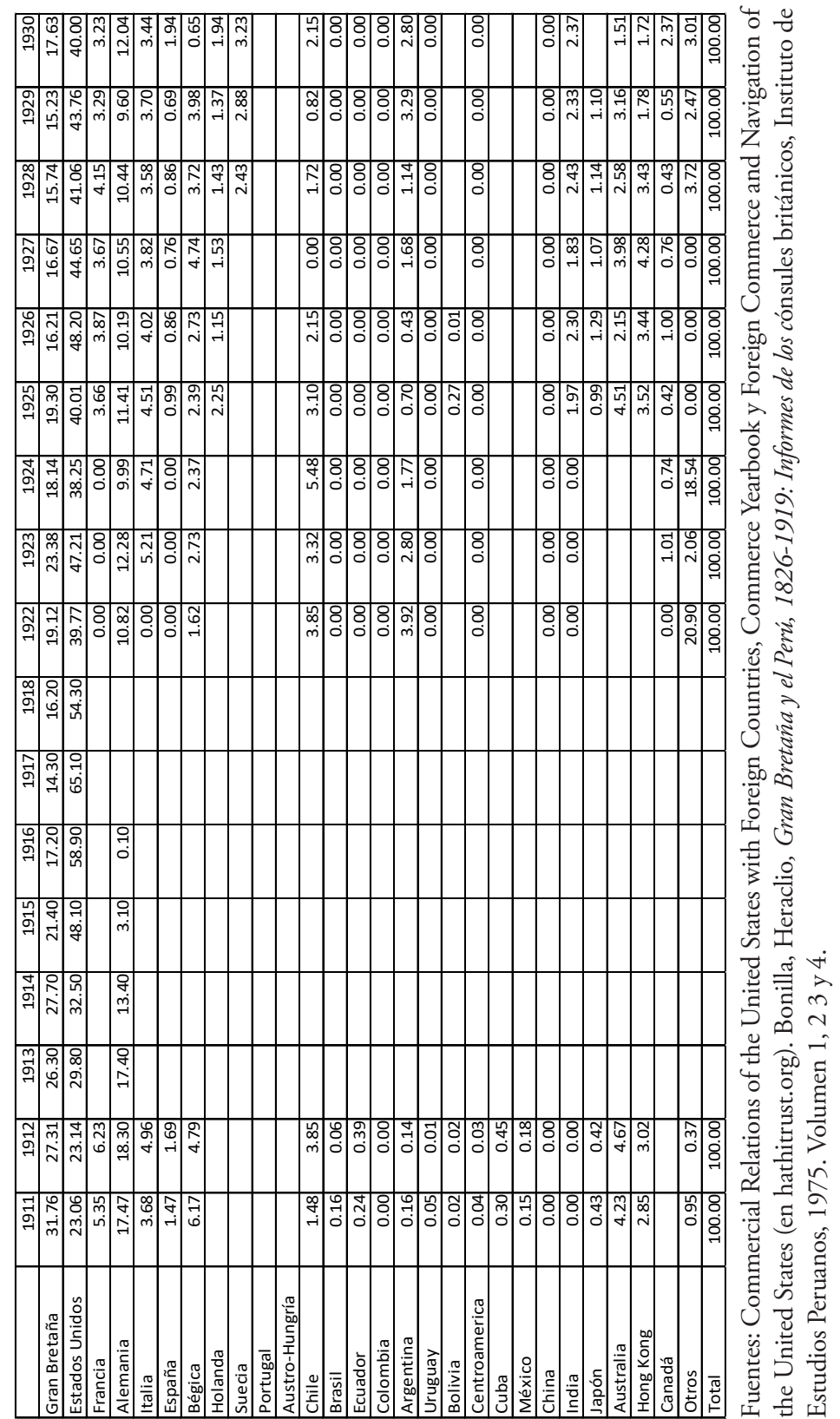




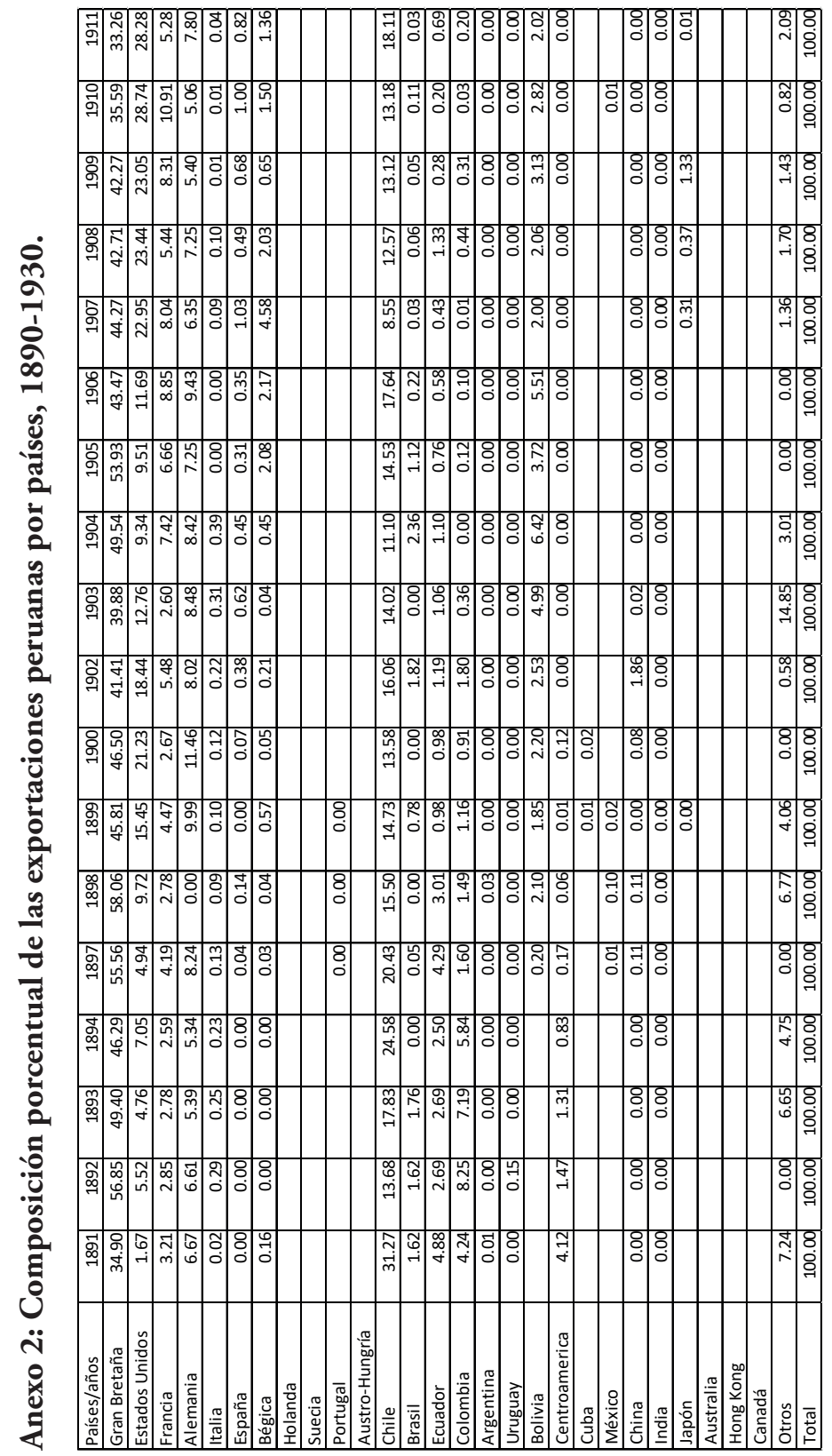




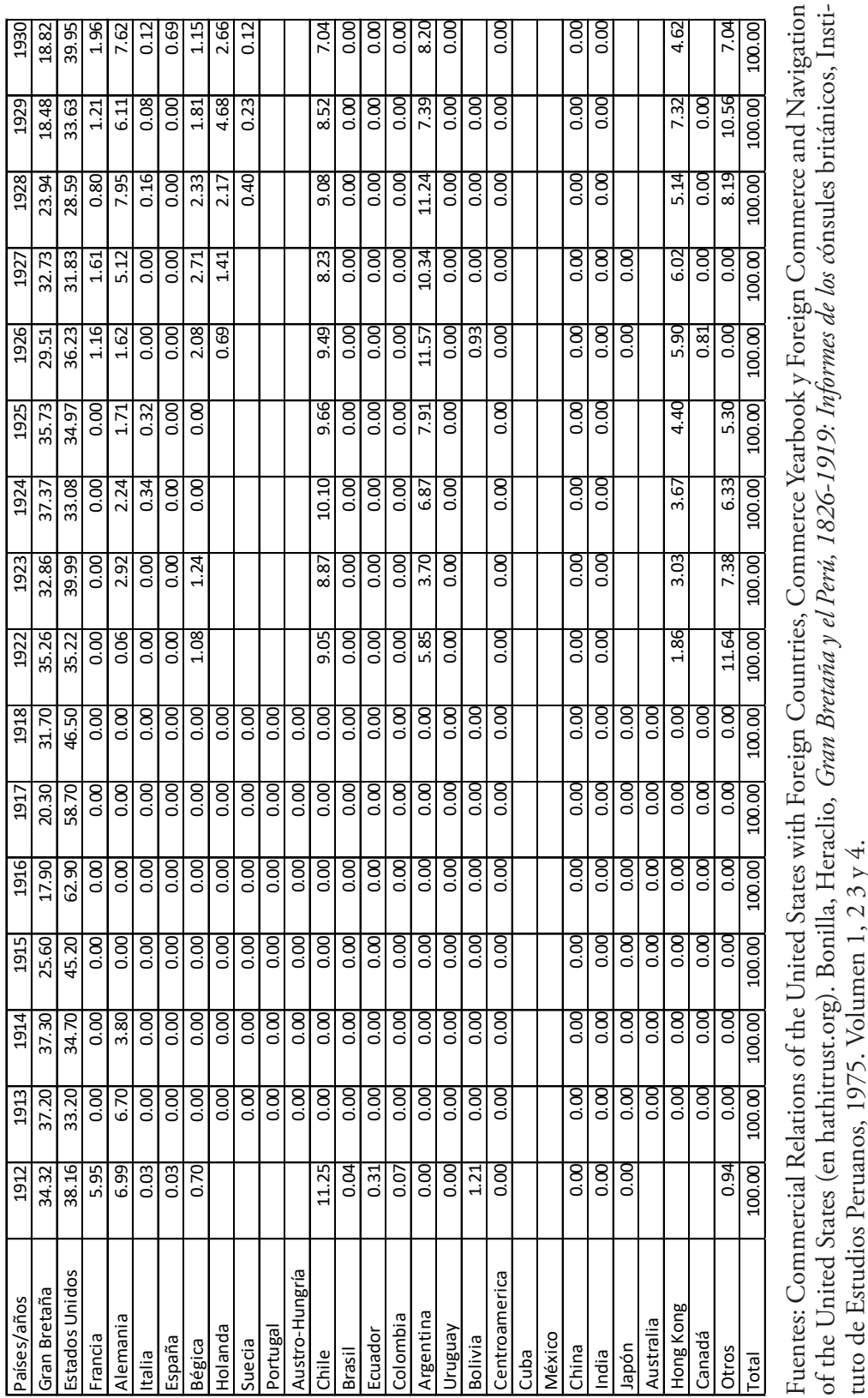




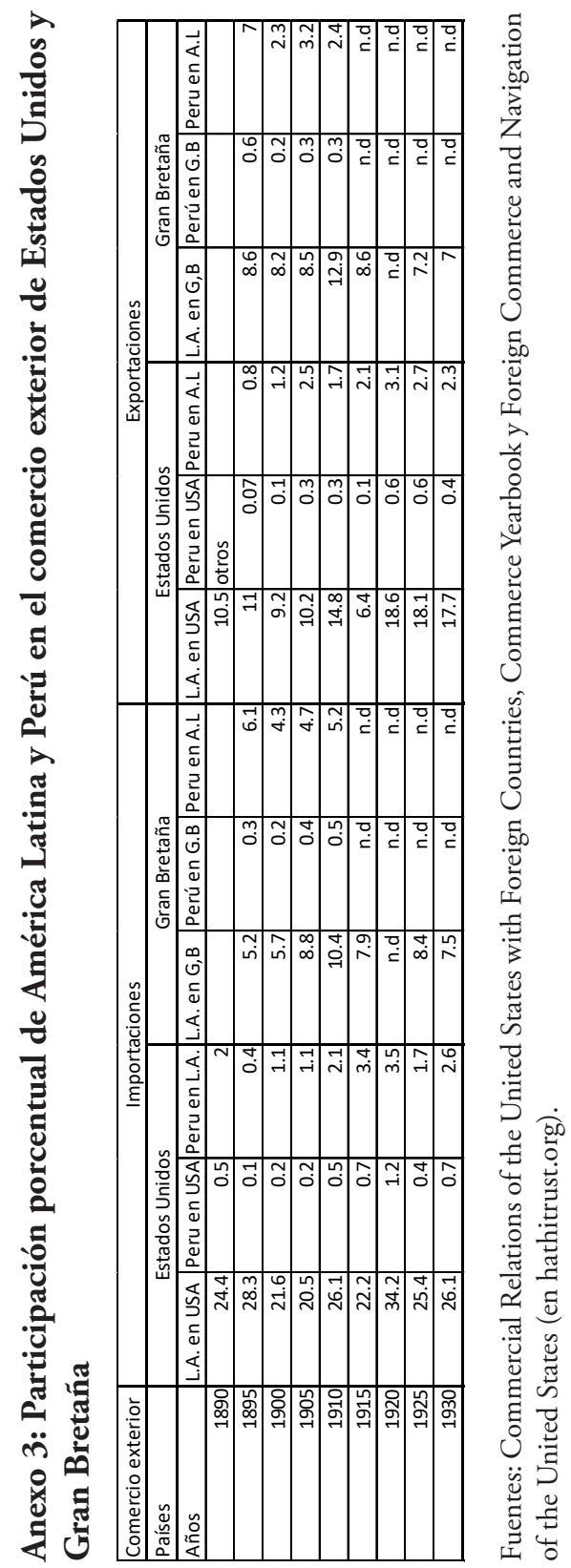

